\title{
RFID-assistierte Stückguterfassung und -handling anhand eines kognitiven Robotersystems
}

\author{
Dipl.-Ing Antonios Bouzakis, Dipl.-Ing Stefan Weigelt, \\ Prof. Dr.-Ing. Ludger Overmeyer \\ Leibniz Universität Hannover \\ Institut für Transport- und Automatisierungstechnik
}

\begin{abstract}
Im Rahmen des durch die Deutsche Forschungsgemeinschaft (DFG) geförderten Forschungsvorhabens „ASEM“ wurde die RFID-Transponderortung für den Einsatz in der Stückgutlokalisierung untersucht. Hierzu wurde mit Transportmodulen eine Lagerhausumgebung nachgebildet und ein Industrieroboter zum Aufnehmen und Palettieren von Stuckgütern mit einem selbstentwickelten Greifersystem ausgestattet. Ein zwischen den Vakuumsaugern des Greifers integriertes RFID-Antennenarray ermöglicht dem Robotersystem in Verbindung mit einer definierten Transponderanordnung auf verschiedenen Musterpaketen Produkterfassungs- und Geometrieerkennungsprozesse ohne einen Werkzeugwechsel für die nachfolgenden Bearbeitungsschritte. Die erreichbare Genauigkeit der Positionserfassung ist in verschiedenen Versuchsreihen insbesondere unter Berücksichtigung der Prozessgeschwindigkeit analysiert worden. Da unterschiedlich große Stückgüter zuverlässig und schnell erfasst werden konnten, ist dieser Prozess besonders für die Verarbeitung von unpalletierter und mit hoher Frequenz anfallender Waren, wie beispielsweise bei der Post oder dem Versandhandel, geeignet.
\end{abstract}

\section{$1 \quad$ Einleitung}

Automatisierte Wareneingangs- und Ausgangsprozesse in der Intralogistik laufen mit immer geringeren Taktzeiten ab. Viele Prozesse der Warenidentifikation und des innerbetrieblichen Materialflusses werden heute bereits mit Hilfe der RadiofrequenzIdentifizierung (RFID) durchgeführt, die aufgrund der flexibleren Einsatzmöglichkeiten zunehmend den Barcode ersetzt. Ein automatisiertes Handling erfordert dabei entweder normale Palletiermaschinen oder kognitive Roboter, die bisher meist von Bildverarbeitungssystemen und Laserscannern unterstützt werden [SKB ${ }^{+} 08$ ], [SWtH05]. In diesem Beitrag wird der Einsatz eines RFID-Systems zur Positionserfassung aufgezeigt. Dabei ermittelt der Roboter die Lage und die Orientierung von Stückgütern, die über einen Rollenförderer in seine Umgebung transportiert werden. Das kognitive Robotersystem besteht aus einem Industrieroboter, ergänzt um handelsübliche RFID-Komponenten. Die notwendigen Positionierungsinformationen werden aus einer Korrelation von in einer Datenbank gespeicherten Geometriedaten und der erfassten Position der TransponderAnordnung auf den Paketflächen ermittelt. 


\section{Stand der Technik}

Die Radiofrequenz-Identifikation (RFID) ergänzt heute auf dem Gebiet der Lagerbestandserfassung mit hohen Ablaufgeschwindigkeiten zunehmend den Barcode. Auch bei der Positionsbestimmung gibt es bereits Ansätze zur Verwendung dieser Technologie, beispielsweise für Navigationssysteme, die auf der Ortung von integrierten RFID-Bodentranspondern im Hochfrequenzbereich basieren. Derartige Anwendungen wurden bereits im Zusammenhang mit fahrerlosen Transportsystemen (FTS) aufgezeigt. Dabei nimmt eine angepasste Anordnung von Antennen die Signalstärke der Transponderantwort auf und berechnet damit eine Abweichung von der geplanten Fahrstrecke [BO09]. In einer weiteren Anwendung wurden ortsfeste RFID-Tags verwendet, um mit der Hilfe von kommerziellen Lesegeräten anhand der erfassten Signalstärke die Entfernung zum Lesegerät zu bestimmen [SW07]. Damit wurde die absolute Lage des Fahrzeugs im Raum bestimmt. Kämpke et al. haben den Einsatz von integrierten Bodentranspondern an zahlreichen Stellen vorgeschlagen, damit ein RFID ausgerüstetes FTS selbständig seine Lage auf dem „Smart Floor“ erkennt [KKPS08]. In Hinblick auf die Verwendung zur Stückguterfassung in einer Lagerhausumgebung wurden zwei RFID- Systeme zuvor bereits auf ihre Funktionsfähigkeit untersucht [BO10a]. Aufgrund des großen Stückgutdurchsatzes sind die Kosten für die Paket-Transponder dabei so gering wie möglich zu halten. Daher wurden Ortungssysteme ausgewählt, die mit handelsüblichen Transpondern arbeiten. Mit zwei unterschiedlichen RFID AntennenSystemen sind Versuche im Hochfrequenzbereich $(13,56 \mathrm{MHz})$ in Verbindung mit verschiedenen Transpondertypen durchgeführt worden. Hierbei handelte es sich zum einen um ein nach ISO 15963-spezifiziertes System der Firma Götting, zum anderen um ein am Institut für Transport- und Automatisierungstechnik (ITA) eigens entwickeltes System nach den Richtlinien der ISO 14443. Den verschiedenen Kombinationen aus Antennensystemen und Transpondern wurden Untersuchungen zur Bestimmung der maximalen Reichweite, Geschwindigkeit und Positioniergenauigkeit unterzogen. $\mathrm{Da}$ die Ergebnisse vielversprechende Möglichkeiten für das Kartonagehandling aufzeigten, wurden verschiedene Ansätze zur Bestimmung der räumlichen Orientierung eines Paketes auf Basis der in die Stückgüter einzubringenden Transponder entwickelt. Der Schwerpunkt bei der Umsetzung des automatisierten Handlingsprozesses lag bei einer zuverlässigen und schnellen Lokalisierung der auf den Stückgütern aufgebrachten RFID-Transponder.

Das für die Untersuchungen in diesem Beitrag ausgewählte System weist neun baugleiche Leseantennen vom Typ FEIG ID ISC.ANT40/30 auf, die jeweils von einem eigenen Lesemodul FEIG ID CPR40.01-UCD gesteuert werden. Dieses Antennenarray ermöglicht eine zweidimensionale Positionsbestimmung der Transponder auf den Kartonoberflächen auf Basis von detektierten Empfangsbereichen mittels eines Scanprozesses [BO10a]. 


\section{$3 \quad$ Lagerhausumgebung}

Nach der Analyse der für die Testanlage notwendigen Anforderungen, wurden ein Industrieroboter sowie ein Fördermodul zur Nachbildung einer Lagerhausumgebung aufgebaut. Dabei wurden logistische Anwendungen berücksichtigt, bei denen ein zu transportierendes Paket die Masse von $15 \mathrm{~kg}$ nicht überschreitet. In diesem Gewichtsbereich fasst ein Produktbehälter grundsätzlich entweder eine kleinere Warenmenge oder nur ein einzelnes Produkt. Der Industrieroboter vom Typ KUKA KR-16 mit 6 Freiheitsgraden wurde ausgewählt, da er eine Nennmaximaltraglast von $16 \mathrm{~kg}$ aufweist. Im Greifbereich des Roboters wurde eine bestehende Förderanlage um einen Rollenförderer RC 27 der Firma Transnorm ergänzt. Dieser Rollenförderer wurde mit Transportrollen der Serie 3500 und einem Steuermodul vom Typ EC300 ausgestattet. Beides wurde von der Firma Interroll zur Verfügung gestellt. Eine Auswahl verschiedener normierter Kartonagen des Versandhandels wurde in den Versuchen als Produktbehälter verwendet. Die Paketgrößen 1, 2, 3 entsprechen den Abmessungen $295 \times 170 \times 125 \mathrm{~mm}, 355 \times 300 \times 150 \mathrm{~mm}$ und $495 \times 325 \times 205 \mathrm{~mm}$. Abbildung 1 zeigt die nachgestellte Lagerhausumgebung.

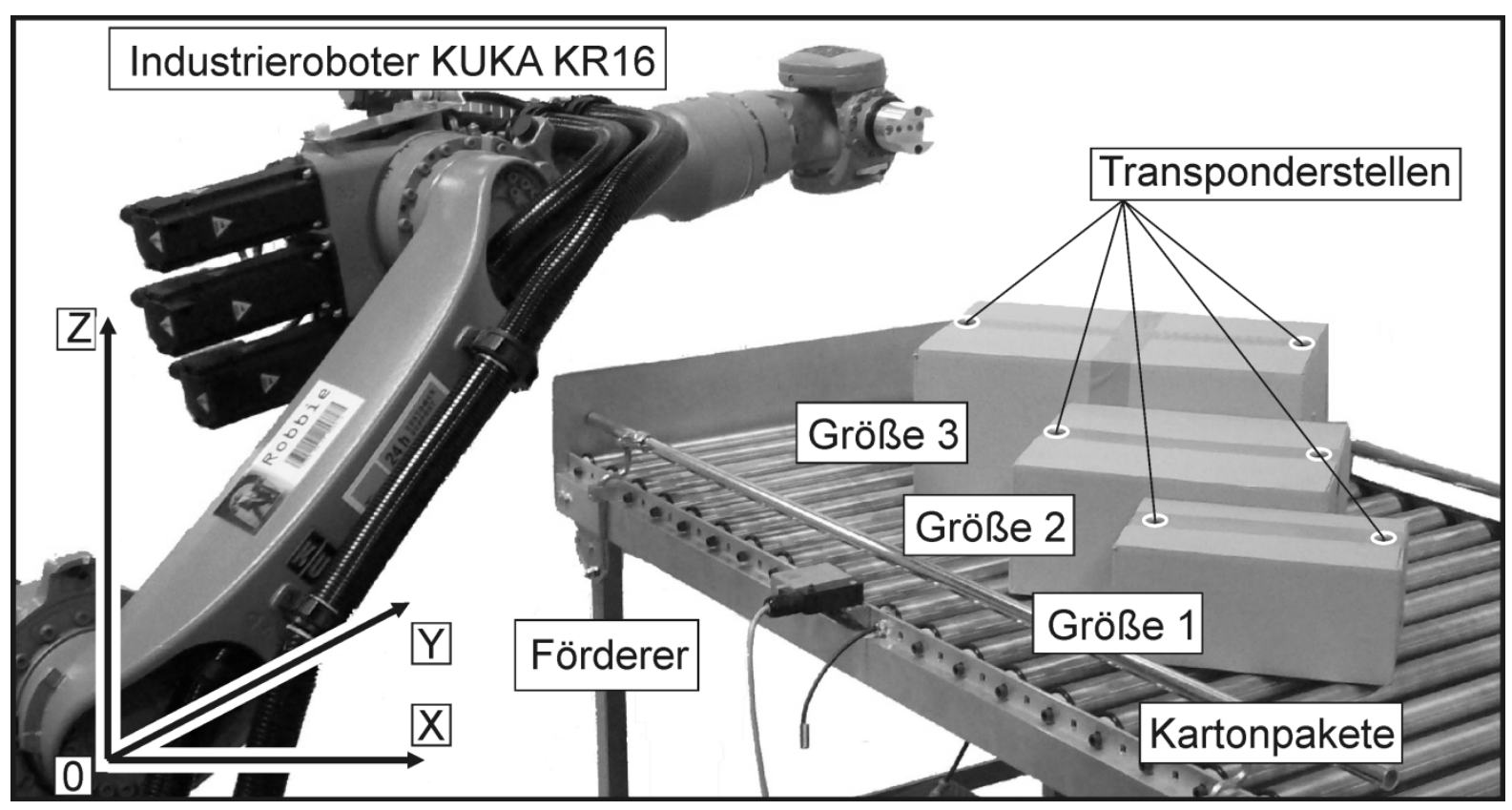

Abbildung 1: Lagerhausumgebung [Quelle: BO10b]

\section{$4 \quad$ Konzeption des Greifsystems}

Gemäß dem aktuellen Stand der Technik wurde ein Greifsystem mit Vakuumsaugern für das Kartonagehandling konzipiert. Nach der Ermittlung von notwendigen Anforderungen für das effiziente Handling unterschiedlicher Paketgrößen, wurde eine Anordnung von 8 Saugnäpfen an einem Aluminiumrahmen montiert. Der Abstand der Saugnäpfe untereinander wurde dabei so gewählt, dass die Stückgüter immer von mindestens 4 Saugnäpfen angehoben werden. Da jeder Saugnapf über eine maximale Saugkraft von $33 \mathrm{~N}$ verfügt, können Pakete bis mindestens $12 \mathrm{~kg}$ sicher angehoben werden. Unter der Berücksichtigung der maximalen Tragfähigkeit des 
Roboterarms von $16 \mathrm{~kg}$ und dem Eigengewicht des kombinierten GreifAntennensystems von $5,5 \mathrm{~kg}$ reduziert sich das maximale Stückgutgewicht auf $10,5 \mathrm{~kg}$. Innerhalb des aufgebauten Greifsystems ist das Antennenarray zwischen den in zwei Reihen angeordneten Saugnäpfen integriert. Ein sonst üblicher Werkzeugwechsel zwischen dem Ortungs- und dem Greifprozess entfällt bei dem hier gezeigten Ansatz. Der genaue Aufbau des kombinierten Ortungs- und Greifsystems ist in Abbildung 2 dargestellt.

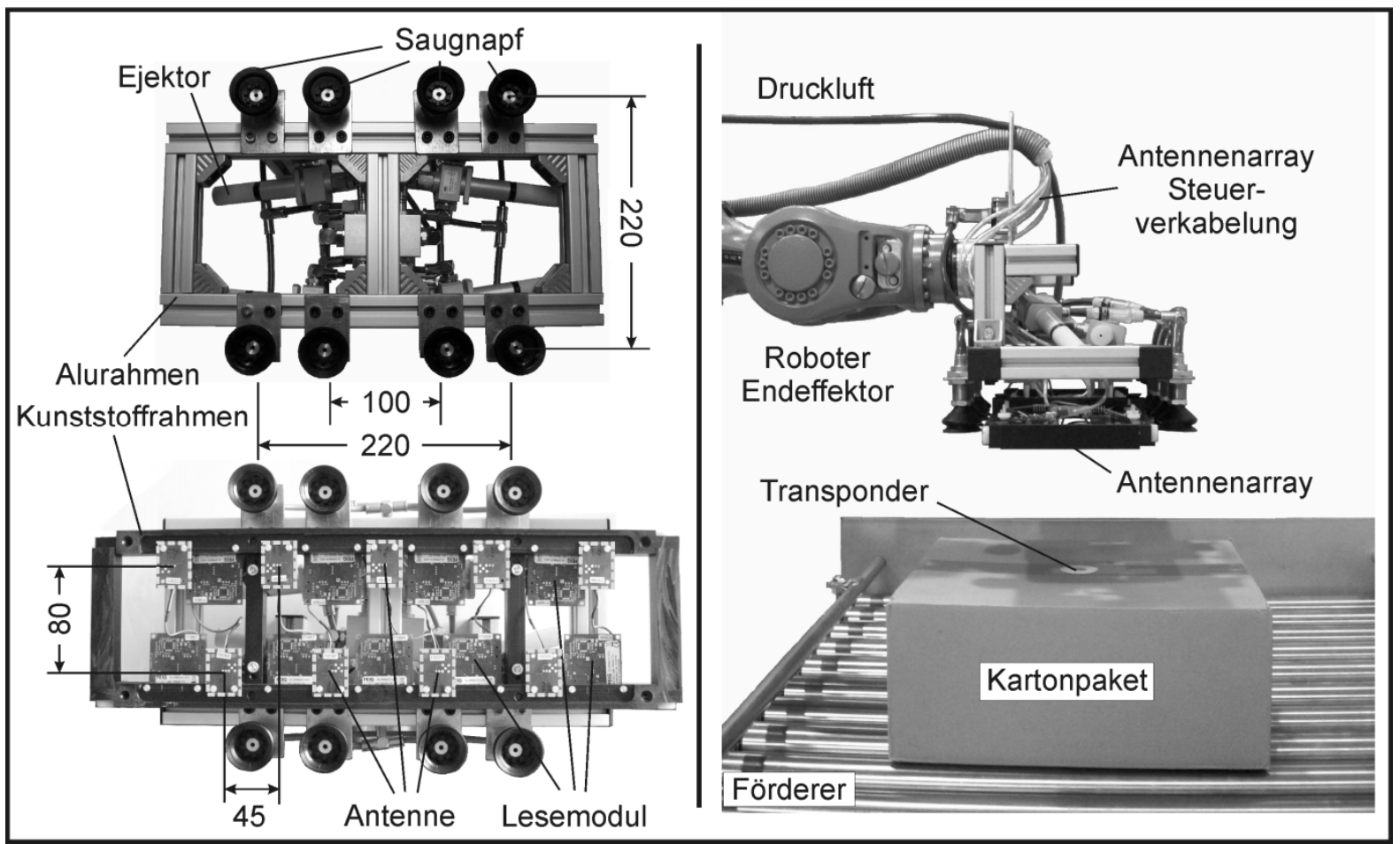

Abbildung 2: Greifsystem mit integriertem RFID-Antennenarray [Quelle: BO10a]

\section{$5 \quad$ Untersuchungen zur Paketerfassung}

Die quaderförmige Paketgeometrie führt zu einer eindeutigen Beschreibung der Lage des Stückgutes auf der Förderfläche durch vier Freiheitgrade. Hierzu gehören die Lagekoordinaten $(x, y, z)$ eines Bezugspunktes auf dem Paket sowie die Drehung a um die Hochachse. Mittels eines Scanprozesses werden 3 der 4 Freiheitsgrade $(\mathrm{a}, \mathrm{x}, \mathrm{y})$ mit Hilfe des Antennenarrays und zwei aufgeklebten, kreisförmigen ISO 14443-Transpondern bestimmt. Diese beiden Transponder sind dabei genau auf einer der beiden Symmetrieachsen der Paketoberfläche in der xy-Ebene und im gleichen Abstand vom Flächenmittelpunkt angeordnet. Die detektierten Empfangsbereiche liefern dadurch nicht nur die $x$ - und $y$-Koordinaten der Transpondermittelpunkte sondern ebenfalls auch den geometrischen Mittelpunkt des Pakets auf der xy-Ebene. Die Drehung a um die Hochachse im Raum wird anschließend aus der Orientierung des Verbindungsvektors zwischen diesen beiden Transpondermittelpunkten bestimmt.

Die Höhe $z$ des auf dem Transportmodul aufliegenden Paketes ist vor dem Scanprozess durch das Antennenarray bereits bekannt, da eine separate 
Leseantenne in einem vorhergehenden Arbeitsschritt die geometrischen Daten des Stückgutes über einen zusätzlichen ISO 15963-Transponder abruft [BO10b]. Der ISO 15963-Transponder, der zwar eine langsamere Ansprechzeit, aber größere Reichweite als die ISO 14443-Transponder aufweist [Fin03], speichert dabei auch weitere für den Materialfluss relevante Daten, wie etwa das Gewicht oder den Inhalt. Desweiteren ist die Ausrichtung der ISO 14443-Transponder auf dem Paket, entweder parallel oder quer zu der längeren Seite des Pakets, hier vermerkt.

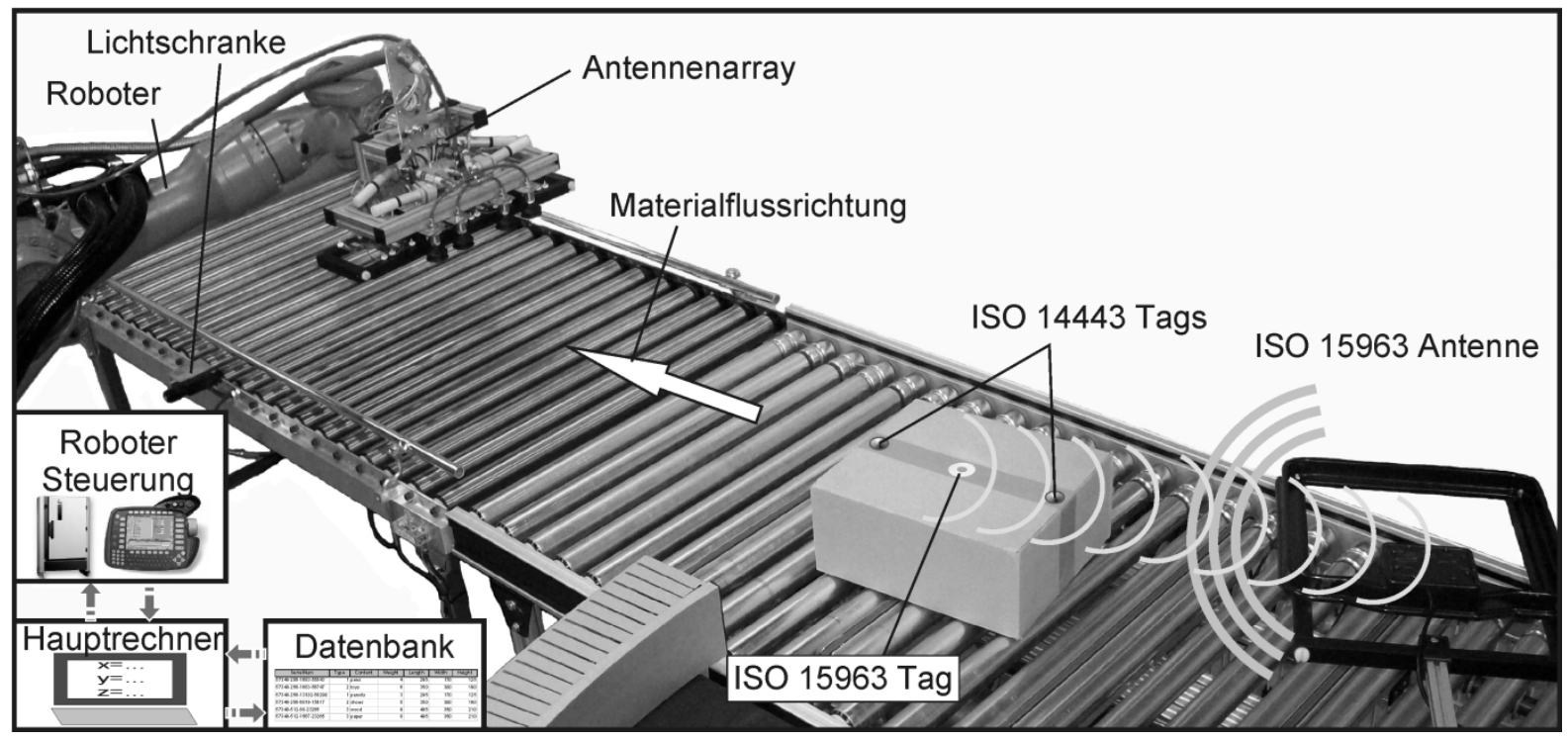

Abbildung 3: Funktionsweise der RFID-assistierten Stückguterfassung [Quelle: BO10a]

Sobald der mittlere ISO 15963-Transponder eines herannahenden Pakets detektiert wird, werden alle gespeicherten Informationen zu einem Hauptrechner gesendet, der das gesamte System steuert. Anhand einer Datenbank werden die übermittelten Daten auf Plausibilität und Vollständigkeit hin überprüft.

Im Rahmen der folgenden Untersuchungen zur Analyse der erreichbaren Genauigkeit bei der Positionsbestimmung wurde das beschriebene Verfahren umgesetzt. Erreicht ein Paket auf seinem Transportweg eine bestimmte Position unterhalb des Greifers, wird der Materialfluss durch das Aktivieren einer Lichtschranke gestoppt. Anschließend verfährt der Roboterarm den montierten Paketgreifer in eine, aus der Stückguthöhe abgeleitete, Position über dem Transportmodul für den nachfolgenden Scanprozess. Zur Bestimmung der Transponderkoordinaten des nun ruhenden Pakets verfährt der Roboter den Paketgreifer mit aktiviertem Antennenarray nach dem folgenden Muster: Zuerst bewegt sich das Ortungssystem entgegen der Transportrichtung des Fördermodules um 700 mm, verschiebt sich danach orthogonal hierzu um 22,5 mm (der Hälfte des Abstands zwischen zwei Antennenelemente) und fährt anschließend wieder zur Ausgangsposition zurück. Dabei werden die durchlaufenen Abschnitte der sich kugelförmig ausbreitenden Empfangsbereiche der beiden ISO 14443-Transponder sowohl bei der Hin- als auch bei der Rückfahrt aufgenommen [BO10a]. 


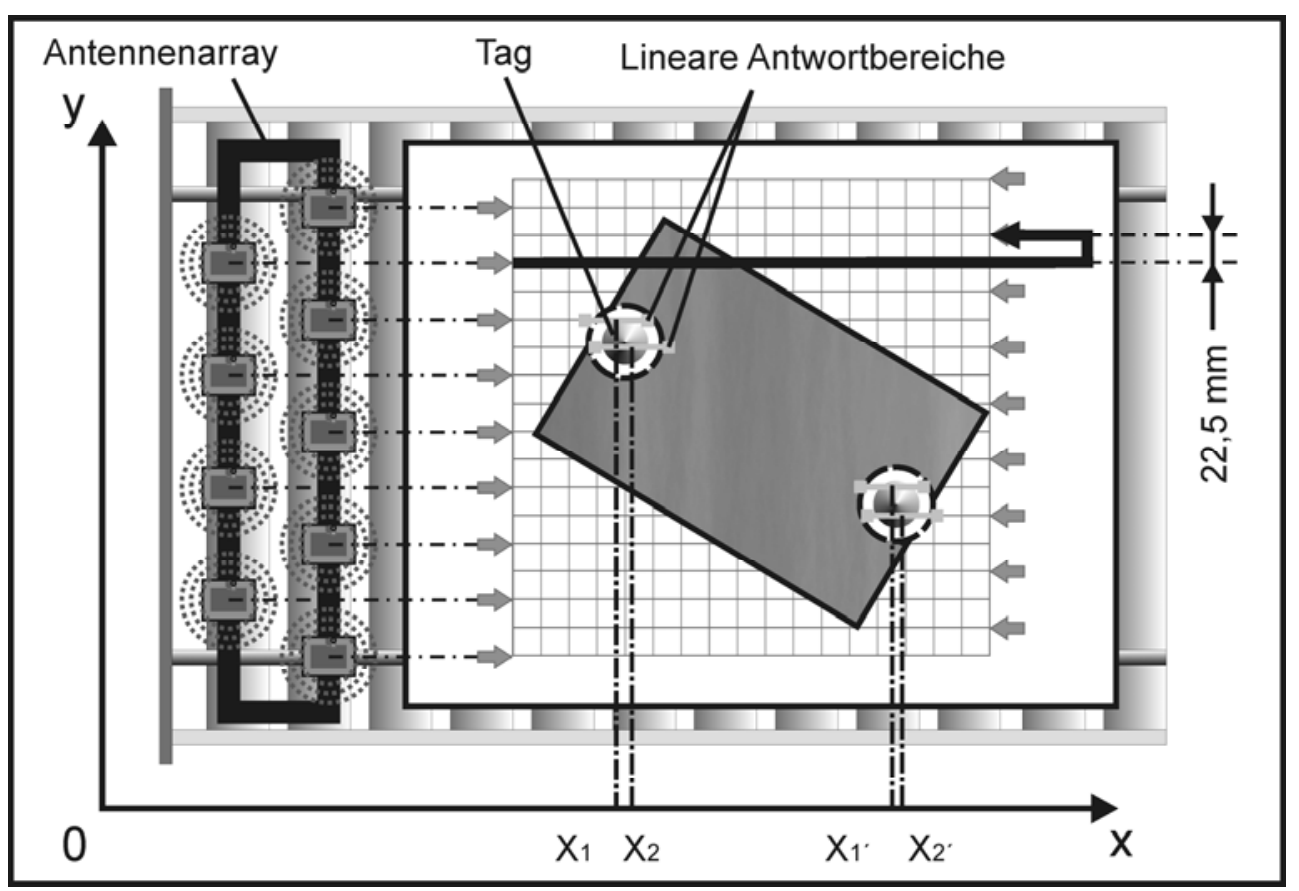

Abbildung 4: Verfahrweg und Berechnung der x-Koordinate

Aus den Mittelpunkten der in Transportrichtung (x-Achse) erfassten Empfangslinien wird auf die Position der Transponder in dieser Richtung geschlossen.

$$
X=\frac{X_{1}+X_{2}}{2}
$$

Mit einem um $90^{\circ}$ in der xy-Ebene gedrehtem Greifer ließe sich mit demselben Verfahren anschließend auch die Position der y-Koordinate des Transponders ermitteln. Um diesen weiteren zeitaufwändigen Scanprozess zu vermeiden, wird in diesem Ansatz die Bestimmung der Transponderposition orthogonal zur Transportrichtung anhand der Anzahl der eingehenden Transponderantworten während der Aufnahme der Empfangslinien in x-Richtung untersucht. Durch die kugelförmige Ausbreitung des Empfangsbereiches wird dieser je nach Versatz der Leseantenne in Bezug auf den zu ortenden Transponder auf der $y$-Achse unterschiedlich lange durchlaufen. In Verbindung mit der gleichbleibenden Frequenz der ausgesendeten Transponderantworten, lässt sich bei zweimaligem Durchfahren des Empfangsbereichs anhand eines Vergleiches der Anzahl der empfangenen Transponderantworten auf die Position des Transponders in y-Richtung schließen. Die Abbildung 5 zeigt die Anzahl der Transponderantworten in Abhängigkeit des vertikalen und horizontalen Abstandes der Leseantenne zu dem Transponder für vier unterschiedliche Scangeschwindigkeiten. 


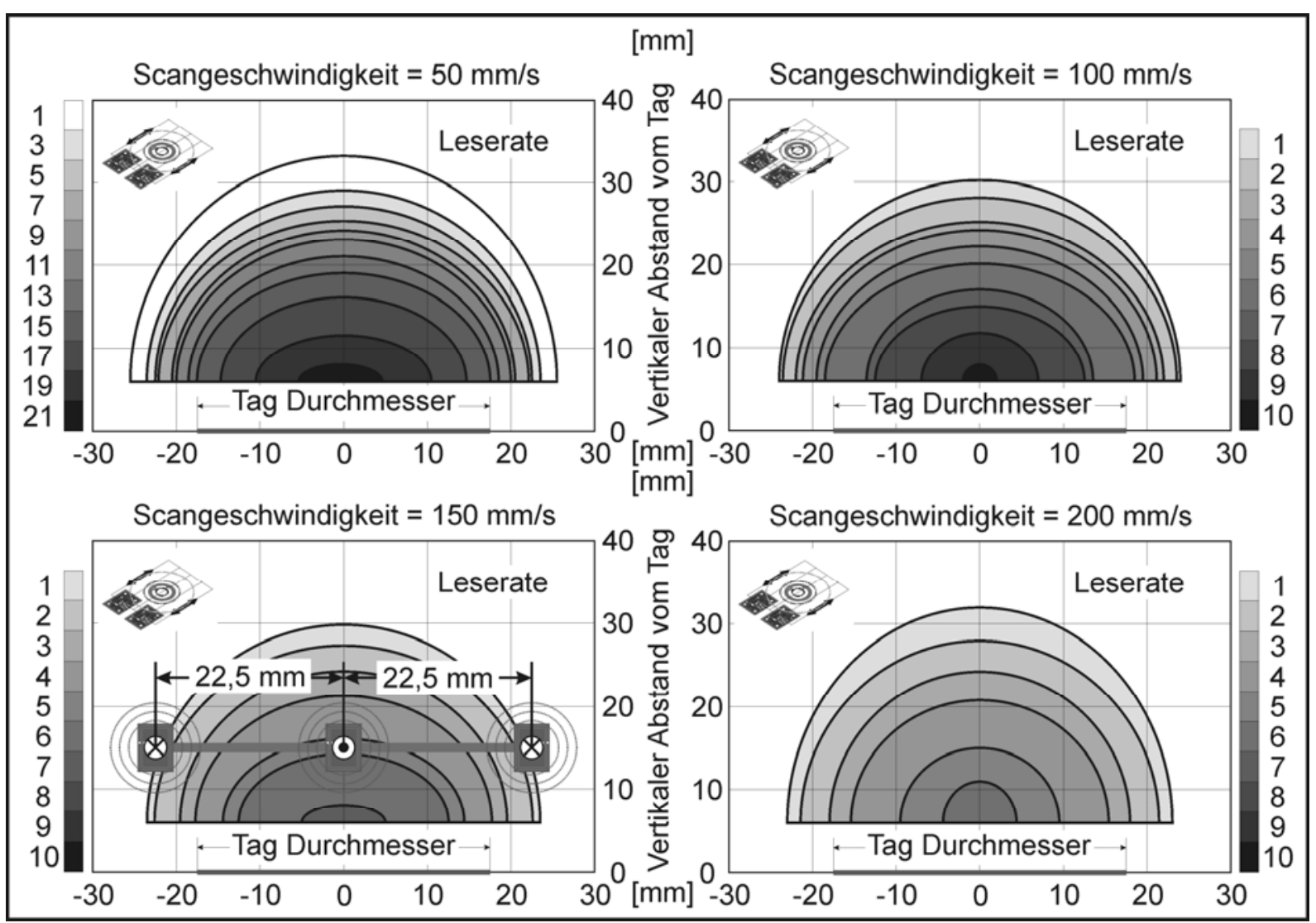

Abbildung 5: Anzahl der Transponderantworten in Abhängigkeit der Position der Leseantenne

Wie in Abbildung 5 unten links dargestellt, ist das zweimalige Durchfahren des Empfangsbereiches bei der Hin- und der um 22,5 mm versetzt stattfinden Rückfahrt des Greifers bis zu einer Höhendifferenz von $15 \mathrm{~mm}$ zwischen Leseantenne und Transponder sichergestellt.

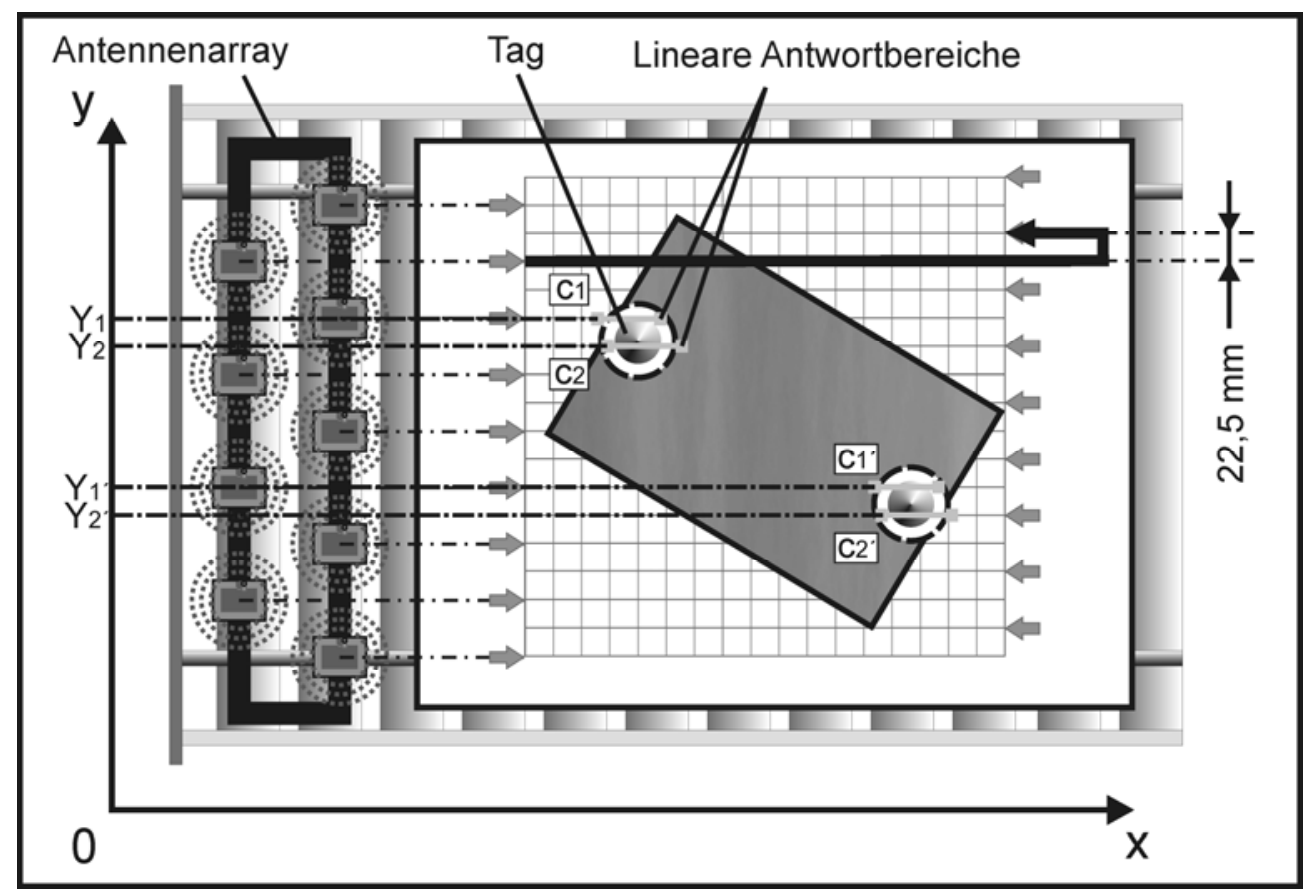

Abbildung 6: Verfahrweg und Berechnung der y-Koordinate 
Mit der Variablen $c$ für die Anzahl der empfangenen Antworten ergibt sich nach Abbildung 6 für die Gleichung zur Bestimmung der y-Koordinate:

$$
Y=\frac{Y_{1} c_{1}+Y_{2} c_{2}}{c_{1}+c_{2}}
$$

Die erreichbaren Genauigkeiten bei der Positionsbestimmung nach dem dargestellten Schema wurden in umfangreichen Versuchsreihen ermittelt. Der Schwerpunkt lag dabei auf der Analyse des Einflusses von Scangeschwindigkeit und Abstand zwischen den Leseantennen und den auf den Paketen aufgebrachten Transpondern.

\section{Ergebnisse}

Zunächst wurde für einen konstanten vertikalen Abstand von $15 \mathrm{~mm}$ zwischen Leseantennen und Pakettranspondern der Einfluss der Scangeschwindigkeit in Bezug auf die Genauigkeit der Positionsbestimmung in x-Richtung untersucht. Die Auswertung der ermittelten Daten ergab, dass bei einer Verfahrgeschwindigkeit von $100 \mathrm{~mm} / \mathrm{s}$ die Standardabweichung $\sigma_{x}$ zur Lokalisierung der x-Koordinate etwa $2 \mathrm{~mm}$ beträgt. Bei einer Geschwindigkeit von $400 \mathrm{~mm} / \mathrm{s}$ liegt $\sigma_{x}$ bereits bei $7,5 \mathrm{~mm}$. Bei Scanprozessen, die mit über $430 \mathrm{~mm} / \mathrm{s}$ durchgeführt worden sind, war eine Erfassung der Empfangslinien teilweise gar nicht mehr möglich. Abbildung 7 stellt die Standardabweichung bei der Positionsbestimmung der x-Koordinate in Abhängigkeit von der Verfahrgeschwindigkeit dar.

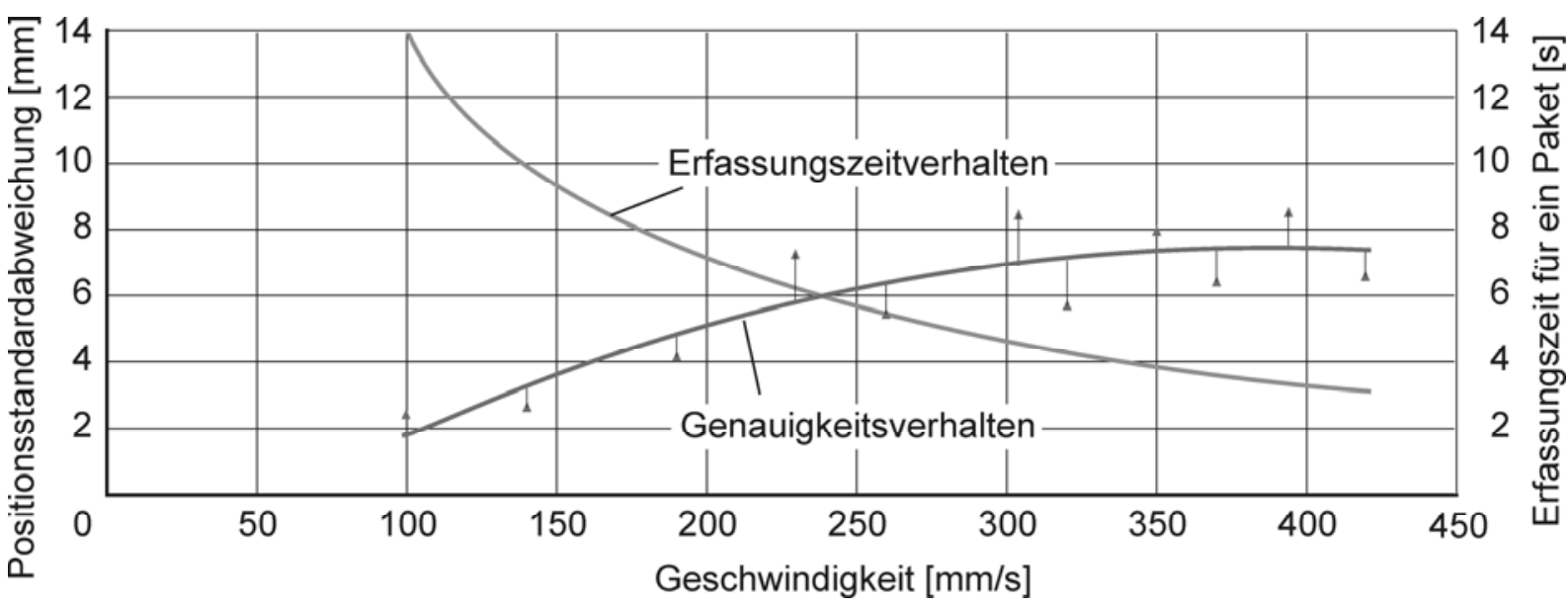

Abbildung 7: Genauigkeits- und Erfassungszeitverhalten für die x-Achse

In Bezug auf die Bestimmung der y-Koordinate wurden Versuche mit unterschiedlichen Verfahrgeschwindigkeiten für die Scanabstände $7,5 \mathrm{~mm}$ und $15 \mathrm{~mm}$ in z-Richtung durchgeführt. Dabei wurden die in der xy-Ebene detektierten Empfangsbereiche in Sektionen mit unterschiedlicher Anzahl von empfangenen Transponderantworten eingeteilt. Eine höhere Scangeschwindigkeit führte dabei zu einer geringeren Anzahl an Antworten über die gesamte Empfangswolke. Wie aus Abbildung 8 hervorgeht, folgt hieraus entsprechend eine geringere Auflösung der Sektionen mit unterschiedlicher Antwortanzahl in y-Richtung. 


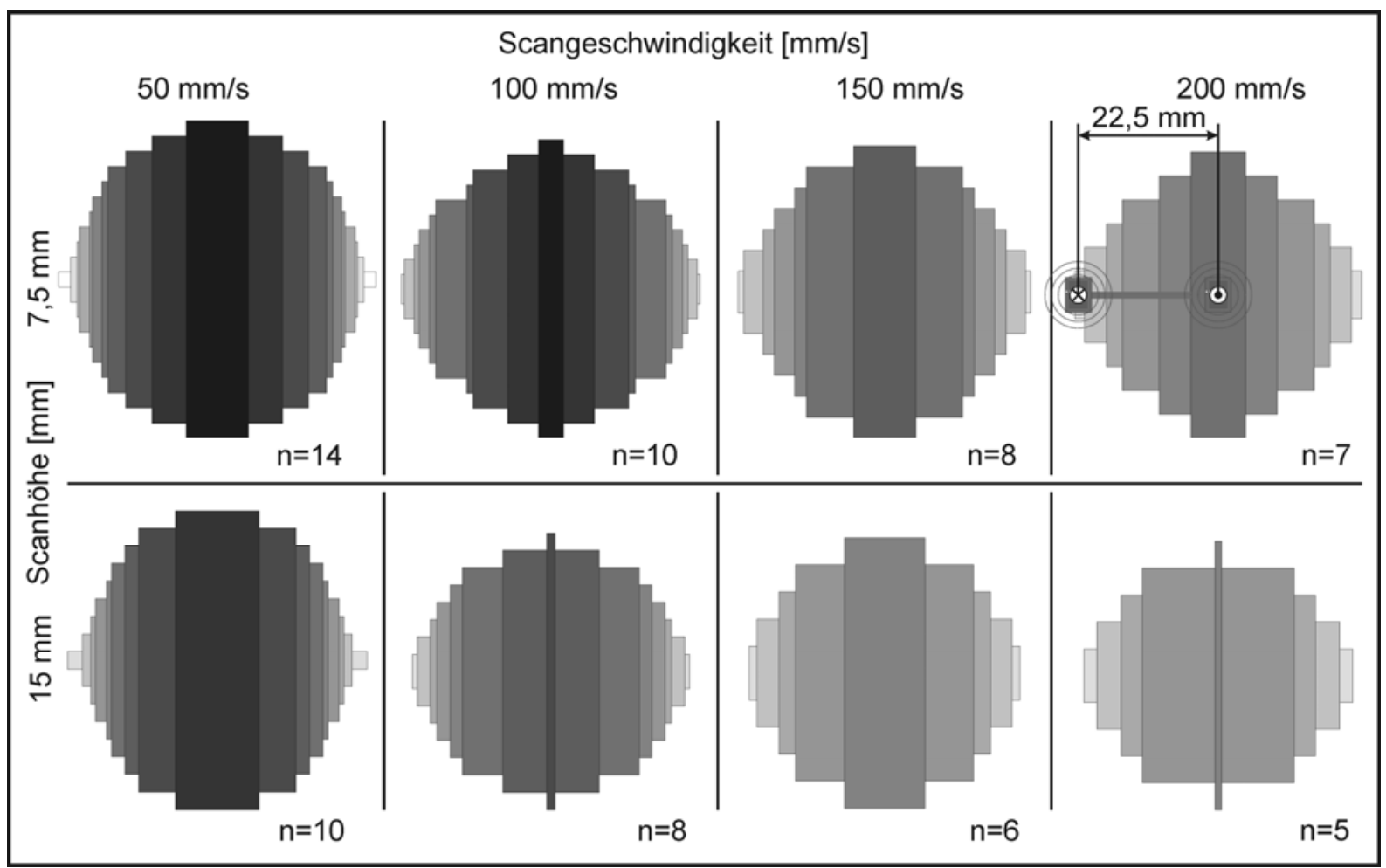

Abbildung 8: Auswertung der Transponderantworten zur Bestimmung der y-Koordinate

Für die ermittelte Aufteilung an Sektionen gleicher Antwortanzahl ergibt sich, unter Berücksichtigung der um 22,5 mm auf der y-Achse versetzt stattfindenden Hin- und Rückfahrt während eines Scanprozesses, die jeweils angegebene Anzahl an möglichen Kombinationen $n$ der durchfahrenen Sektionen. Unter der idealisierten Annahme, dass die Sektionen gleicher Antwortanzahl innerhalb des Empfangsbereiches die gleiche Ausdehnung in y-Richtung aufweisen, ergibt sich für die maximale Positionsabweichung der y-Koordinate:

$$
\Delta Y_{\max }=\frac{22,5 \mathrm{~mm}}{n}
$$




\section{$7 \quad$ Zusammenfassung und Ausblick}

Die in der Intralogistik gewünschten geringen Taktzeiten erfordern einen möglichst schnellen Ablauf von Stückguterfassungs- und handlingsprozessen. In dem beschriebenen Ansatz wurde eine Lagerhausumgebung nachgebildet, in der ein Roboter ohne einen Werkzeugwechsel die Geometrie von Stückgütern erfassen und diese anschließend direkt weiteren Prozessen des innerbetrieblichen Materialflusses zuführen kann. Hierzu wurde der Roboterarm mit einem selbst entwickelten Greifersystem mit integriertem Antennenarray zur Positionsbestimmung mittels Radiofrequenz-Identifikation (RFID) ausgestattet. Auf der Oberfäche von Musterpaketen in normierten und häufig anzutreffenden Größen wurden handelsübliche HF-RFID-Transponder angebracht. Die mit diesem System erreichbare Genauigkeit wurde am Beispiel zweier unterschiedlicher Verfahren zur Ermittlung der Lagekoordinaten, in Transportrichtung sowie orthogonal der Transportrichtung, aufgezeigt. Insbesondere wurde auch die Abhängigkeit der Geschwindigkeit des Scanprozesses auf die erreichbare Präzision untersucht. Im Gegensatz zu konventionellen Handlingsysteme ist bei der aufgezeigten Lösung keine vorherige Zwangsorientierung der Stückguter notwendig, wodurch das System sehr flexibel einsetzbar ist. Desweiteren arbeitet es schneller als übliche Laserscanner und ist wesentlich preiswerter $\mathrm{zu}$ realisieren als ein Bildverarbeitungssystem. Zudem ist die RFID-Transponderortung sehr robust. 


\section{Literatur}

[BO09] Baum, M.; Overmeyer L. (Hrsg.): Transpondergestützte Fahrzeugleitsysteme. Hannover: Dissertation, Leibniz Universität Hannover, Berichte aus dem ITA, Band 01/2009, 2009.

[BO10a] Bouzakis, A.; Overmeyer L.: RFID-Assisted Detection and Handling of Packages. ROMANSY 18 Robot Design, Dynamics and Control - CISM Courses and Lectures, vol. 524, ISBN 978-3-7091-0276-3. Wien, New York: Springer, 2010.

[BO10b] Bouzakis, A.; Overmeyer L.: Package Localization in a Warehouse Environment by Means of RFID. In: at-automatisierungstechnik, 7/2010, ISSN 0178-2312. München: Oldenbourg Verlag, 2010.

[Fin03] Finkenzeller, K.: RFID-Handbook - Fundamentals and Applications in Contactless Smart Cards and Identification, 2nd edition, Southern Gate, England: Wiley \& Sons, 2003.

[KKPS08] Kämpke, T.; Kluge, B.; Prassler, E.; Strobel, M.: Robot Position Estimation on a RFID-Tagged Smart Floor. In: Field and Service Robotics, Band 42/2008, ISBN 978-3-540-75403-9. Berlin, Heidelberg: Springer, 2008.

[SKB ${ }^{+}$08] Scholz-Reiter, B.; Kirchheim, A.; Burwinkel, M.; Echelmeyer, W.; Rhode, M.; Schmidt, K.: Automatische Entladung von Stückgütern durch ein kognitives Robotersystem. In: Industrie Management 24/2008. Berlin: Gito mbH Verlag für Industrielle Informationstechnik und Organisation, 2008.

[SW07] Md. Suruz, M.; Wail, G.: An RFID-Based Robot Navigation System with a Customized RFID Tag Architecture, Proceedings of the IEEE International Conference on Microelectronics. Kairo, Ägypten: 29-31. Dezember 2007.

[SWtH05] Schmidt, T.; Wohlfahrt, A.; ten Hompel, M.: Greifertechnologie im Materialfluss, In: Hebezeuge und Fördermittel, 5/2005, S. 82-85. Berlin: Huss-Medien $\mathrm{GmbH}, 2005$. 